Ann. Abeille, I964, 7 (I), 47-53.

\title{
UN DISPOSITIF ASSURANT LA SUPPRESSION TOTALE DU PILLAGE ET DE L'AGRESSIVITÉ DES ABEILLES PENDANT LES VISITES DES RUCHES
}

\author{
J. FRESNAYE \\ Station expérimentale d'Apiculture, \\ Centre de Recherches agronomiques du Sud-Est, Montfavet (Vaucluse)
}

SOMMAIRE

L'auteur donne la description d'une cabine facilement démontable et transportable, qui assure la suppression totale du pillage et de l'agressivité des abeilles pendant les visites des ruches. Ce dispositif est composé d'un parasol et d'un tulle, l'ensemble formant un abri qui empêche les abeilles pillardes de s'attaquer à la ruche visitée. Cette cabine-parasol serait à conseiller particulièrement pour la conduite des abeilles très agressives. Les essais effectués démontrent sa complète efficacité. Il semble, en conséquence, que l'agressivité des abeilles pendant les visites soit principalement due au réflexe de défense de la colonie contre les abeilles pillardes venues des ruches voisines.

\section{INTRODUCTION}

L'agressivité des abeilles, de même que le pillage, sont deux fléaux de l'apiculture bien connus des praticiens. La présence de l'un de ces deux phénomènes entraîne d'ailleurs, bien souvent, la manifestation de l'autre. Les races d'abeilles très agressives sont généralement très pillardes. L'apparition du pillage augmente l'agressivité dans des proportions considérables (LECOMTE I96I) et l'on peut dire que ces deux phénomènes s'amplifient mutuellement. Leur déclenchement se produit, en général, plus facilement lorsque l'on ouvre les ruches pour les différents travaux apicoles et l'apiculteur en est rapidement averti car il en est la première victime.

La fumée obtenue par la combustion lente de produits variés reste la seule défense de l'homme contre l'agressivité de 1'abeille. L,es quelques essais de produits chimiques ainsi que l'emploi des bombes à aérosols contenant des fractions actives de la fumée n'ont pas apporté de résultats convaincants.

Nous avons envisagé la possibilité de réduire le pillage pendant les visites à l'aide 
d'une cage dont les parois grillagées empêcheraient les abeilles étrangères de venir attaquer la ruche ouverte. Les premiers essais furent encourageants, hormis l'impossibilité presque totale de l'utiliser pour un grand nombre de ruches, en raison des difficultés de déplacement du matériel.

L'idée d'utiliser une cage contre le pillage et l'agressivité des abeilles n'est pas nouvelle. WeBER (I943), cité par Niguille (I947), a construit un appareil portatif qui, fixé comme une hotte au moyen de bretelles sur le dos de l'apiculteur, permet de travailler à l'abri des attaques des abeilles étrangères à la ruche visitée. Il est constitué principalement d'un voile de mousseline cylindrique à l'intérieur duquel 1'apiculteur peut travailler. Les abeilles qui s'échappent de la ruche visitée peuvent sortir à l'extérieur grâce à des orifices situés en haut du cylindre. WEBER assure que l'usage de la fumée devient totalement inutile en raison du calme parfait des abeilles.

La douceur des abeilles sous cage est par ailleurs signalée par Chauvin (I953), mais LECOMTE (I955) considère que 1'agressivité est normale dans les grandes serres.

La méthode de WEBER ne fit que peu ou pas d'adeptes et l'article repris par la revue " l'Apiculteur " en I947 ne semble pas avoir donné lieu à des applications pratiques.

Nous nous sommes demandés quelles pouvaient être les raisons de l'oubli de cette méthode et il nous a semblé que les plus motivées étaient d'une part le harnachement qui gêne et fatigue l'apiculteur et, d'autre part, l'impossibilité de visiter des ruches posées sur le sol ou sur des socles très bas. En effet, en se baissant, on entraîne l'appareil dans son mouvement et il devient impossible de travailler. L,e dispositif n'est donc utilisable que par l'amateur qui a quelques colonies. Encore faut-il qu'elles soient disposées sur des socles permettant de travailler sans se baisser.

C'est pourquoi nous avons cherché à améliorer notre cage et notamment sous le rapport de la maniabilité. C'est ce qui nous a conduits à la réalisation de la "cabineparasol ".

\section{MATÉRIEL, ETT TECHNIQUE}

L'armature et le toit du dispositif sont constitués par un grand parasol de I,60 à $1,70 \mathrm{~m}$ de diamètre. Des parasols plus petits pourraient être utilisés, mais on ne pourrait alors plus travailler à deux personnes ni surtout avoir à portée de la main le matériel nécessaire. Le parasol doit être choisi de couleur très claire. Le blanc serait le mieux, mais ne se trouve pas facilement dans le commerce. On trouve, par contre, des jaunes clairs qui peuvent très bien convenir. La hampe du parasol doit être suffisamment longue pour que l'on puisse aisément se tenir debout sous celui-ci lorsqu'il se repose sur le sol. A l'extrémité de chacune des baleines, à l'extérieur, des anneaux inoxydables sont fixés. Ils soutiennent le tulle qui constitue la paroi de la cabine. Ce tulle doit être blanc. Il a une hauteur légèrement inférieure à la distance comprise entre le sol et le bord du parasol, soit environ $\mathrm{I}, 50 \mathrm{~m}$ à $\mathrm{I}, 7 \mathrm{O} \mathrm{m}$ suivant la taille des utilisateurs. Il est accroché au parasol par l'intermédiaire de crochets qui laissent un espace de 4 à $6 \mathrm{~cm}$ vers le haut et qui sont facilement placés ou enlevés. L'espace ménagé entre le bord du parasol et le haut du tulle servira de sortie aux abeilles qui s'échappent de la ruche et qui trouvent très facilement cette sortie placée à la partie la plus élevée de la zone lumineuse. Le tulle, dont la longueur sera égale à la circonférence du parasol, soit $5,34 \mathrm{~m}$ pour un diamètre de $1,7 \circ \mathrm{m}$, peut être cousu dans sa hauteur pour former un cyclindre sous le parasol. Il faudra alors le soulever pour pénétrer dans la cabine. Il est donc préférable de le fermer à l'aide d'une grande fermeture " Eclair " ou de tout autre système permettant une ouverture rapide et facile. A la base du tulle il convient de fixer des masses de plomb qui tendent la cage et évitent que le vent ne la déplace trop facilement. On pourrait également prévoir un cercle métallique qui serait fixé à la place des masses de plomb, mais ce dispositif manque de commodité.

Pour tenir debout, la cabine doit être fixée à la ruche. Le plus pratique est d'utiliser un serre- 
joint de menuisier que l'on choisira de dimension suffisante pour qu'il serre la ruche dans son sens le plus long. Pour les ruches Dadant et Langstrotir, un serre-joint de $55 \mathrm{~cm}$ convient très bien. A l'extrémité du serre-joint, du côté de la mâchoire fixe et perpendiculairement à celle-ci, on soude un tube métallique de $10 \mathrm{~cm}$ de long environ et de diamètre intérieur légèrement plus grand que le diamètre de la hampe du parasol.

Pour monter la cage, on commence par fixer le serre-joint le plus haut possible sur le corps de la ruche à visiter. On glisse la hampe du parasol dans le tube soudé au serre-joint et l'on enfonce légèrement dans le sol la pointe de la hampe. On obtient ainsi un socle idéal constitué par la ruche qui pèse de 25 à fo kg, donc très stable, et qu'il est inutile de déplacer puisque chaque ruche devient socle pour sa propre visite. Il ne reste plus cu'à suspendre le tulle aux anneaux par ses crochets et la cabine est montée (fig. I et 2). Dans un rucher, il n'est nul besoin de démonter la cage pour changer de ruche. Il suffit de soulever assez haut le parasol en le tenant par la hampe, de changer le serre-joint de ruche et de remettre la hampe dans le tube. Cette opération ne prend que quelques secondes.

\section{RÉSULTATS}

Les observations concernant l'efficacité de la cabine parasol ont été faites accessoirement pendant les travaux apicoles. Aucun essai systématique n'a été préparé, mais l'efficacité du dispositif est telle que nous pensons utile de publier nos résultats.

La Station expérimentale d'Apiculture de Montfavet utilise généralement la race d'abeille noire (Apis mellifica mellifica) pour ses diverses expériences. Sans être d'une très grande agressivité, cette abeille est cependant loin d'être aussi douce que l'abeille caucasienne ou l'abeille carniolienne. Ajoutons que nos expériences nous amènent bien souvent à faire subir à nos ruches, par tous les temps, des traitements asse $z$ rudes.

Les occasions d'utiliser la cabine-parasol sont done fréquentes, mais nous ne décrirons que trois cas précis où son utilisation a rendu les plus grands services.

\section{$1^{\text {re }}$ obseriation.}

A la fin d'octobre I962, il nous fallait visiter 25 ruchettes I)ADANT 5 cadres, peuplées d'essaims dans lesquels de jeunes reines avaient été introduites I5 jours plus tôt. Il fallait vérifier la présence de la reine et mesurer la surface du couvain. Une vingtaine de fortes ruches complètaient le rucher.

Le I er jour, nous faisons une tentative de visite sans cabine-parasol. Il fait beau temps, mais le vent est frais et les abeilles ne peuvent trouver aucune provende aux alentours. Au moment où nous visitons la troisième ruchette le pillage se déclenche et, à la cinquième, nous sommes obligés de renoncer à poursuivre le travail, le risque de perdre les jeunes colonies étant trop important. Nous réduisons les entrées des ruches et des ruchettes au maximum et arrosons copieusement les alentours avec un jet d'eau pour calmer l'effervescence. Nous pourons donc considérer qu'il était impossible de visiter longuement les ruchettes sans risquer de voir notre expérience complètement anéantie.

Le $2^{e}$ jour, au même moment de la journée que la veille, nous installons notre cabine-parasol et commençons nos mesures. Avec de très légères bouffées de fumée, nous tenons les abeilles en respect sans difficulté. Pendant $2 \mathrm{~h} 30$, nous visitons I 7 ruchettes, chacune d'elles restant ouverte 8 à Io minutes pour les besoins de nos observations. A ce stade de notre travail, nous n'avons encore reçu aucune piqûre. 
Les abeilles sont parfaitement calmes. Après les visites, les ruches refermées conservent leur sérénité et aucune pillarde ne vient les attaquer. Il n'y a aucune activité anormale à l'entrée des fortes colonies voisines. Ayant fait ces diverses constatations, nous replions notre cabine-parasol et nous visitons les 3 ruchettes restantes. Bien que la visite de ces colonies ne dure que 25 minutes environ le pillage se déclenche et le nombre de piquires s'accroît très vite. Nous sommes de nouveau dans l'obligation de réduire les entrées de ruches.

On peut donc affirmer que, sans notre cabine-parasol, il aurait été impossible de terminer nos relevés.

$2 \mathrm{e}$ observation.

A la fin de mai 1963 nous commençons un élevage de reines dans Io ruches, dans un rucher situé dans les Alpilles. A cette époque de l'année les abeilles ne trouvent plus de nectar et il devient difficile d'ouvrir les ruches sans provoquer le pillage. Or, pour le travail prévu, il fallait rechercher la reine de chaque ruche éleveuse et 1 'isoler, ce qui est assez long, bien que toutes nos reines soient marquées. Nous tentons de faire notre travail sans la cabine-parasol, mais le pillage s'installe très vite et, pour éviter que nos puissantes colonies éleveuses ne se massacrent les unes les autres ou n'attaquent d'autres ruches moins fortes, nous sommes dans l'obligation de renoncer à entreprendre notre élevage de reines ce jour-là. Ilès le lendemain, nous installons notre cabine-parasol et nous pouvons faire tout notre travail sans aucune difficulté et sans piqûres.

\section{3e observation.}

On sait que les abeilles marocaines (Apis mellifica intermissa) sont particulièrement agressives. Certains travaux apicoles, tels que la recherche de la reine, deviennent pratiquement impossibles du fait de cette agressivité quasi maladive. I.orsqu'on ouvre une ruche de cette race, les abeilles se précipitent sur toutes les personnes situées dans un rayon de 30 mètres et le nombre des piqûres est très important.

Or, dans le courant de juillet Ig63, nous devions prélever des mâles dans une ruche d'abeilles marocaines pour inséminer artificiellement une dizaine de reines de la même race. Ce n'est pas sans une certaine appréhension que nous nous préparions à rechercher les mâles adultes dans la ruche surtout lorsque l'on sait qu'il est nécessaire de revenir à la ruche fréquemment, environ toutes les Io minutes, pour prélever de nouveaux mâles. Nous avons eu alors la preuve de l'efficacité de la cabineparasol. Pendant 2 heures, nous avons pu ouvrir constamment la même ruche et la bouleverser de fond en comble, sortant tous les cadres pour rechercher les mâles, sans recevoir une seule piqûre et en utilisant un minimum de fumée.

Ces quelques observations choisies parmi beaucoup d'autres aussi probantes, montrent la pleine efficacité de la cabine-parasol.

En apiculture pratique, la cabine-parasol trouvera son emploi dans les recherches de reines, l'examen de l'état du couvain, le dépistage des maladies, l'évaluation des provisions, l'élevage des reines et même, dans certains cas, la récolte du miel. La production de la gelée royale, qui exige un travail à date fixe quelles que soient les conditions météorologiques, se trouvera grandement facilitée. La cabine-parasol pourra alors servir éventuellement de protection contre la pluie. 

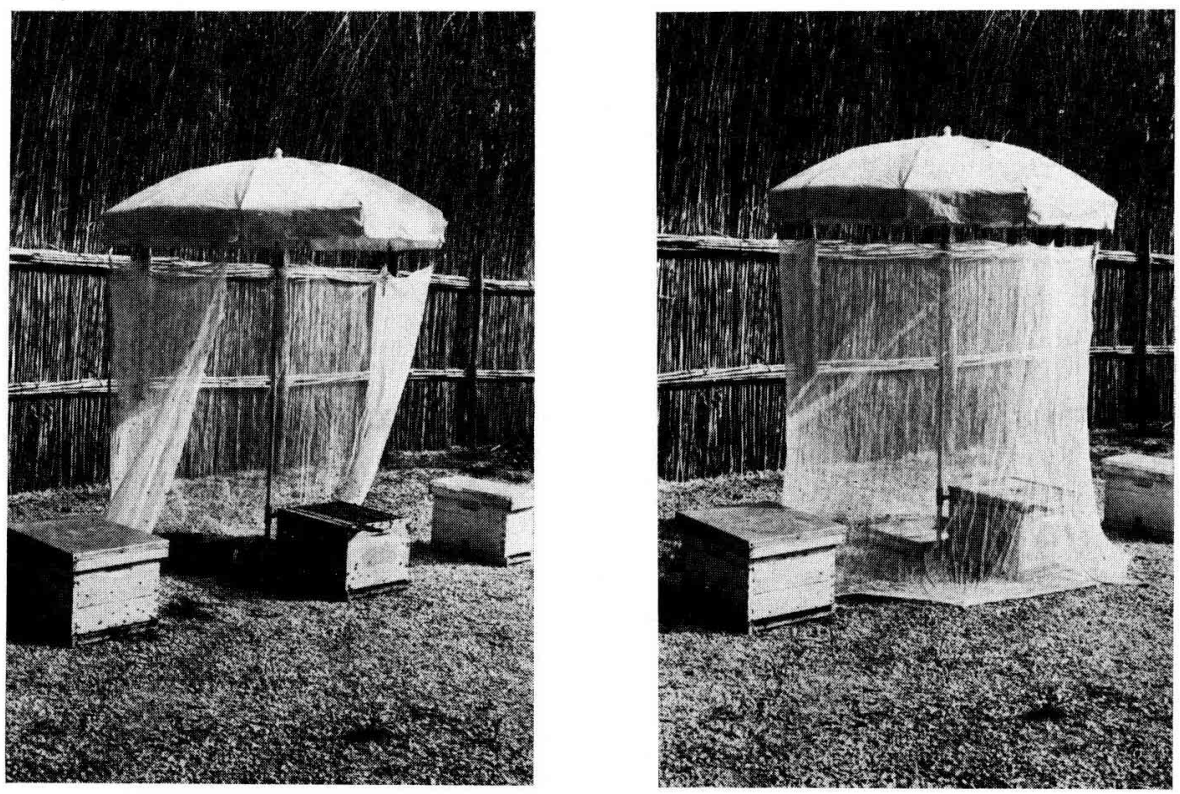

Fig. 1. et Fig. 2. - Monlage de la cabine-parasol

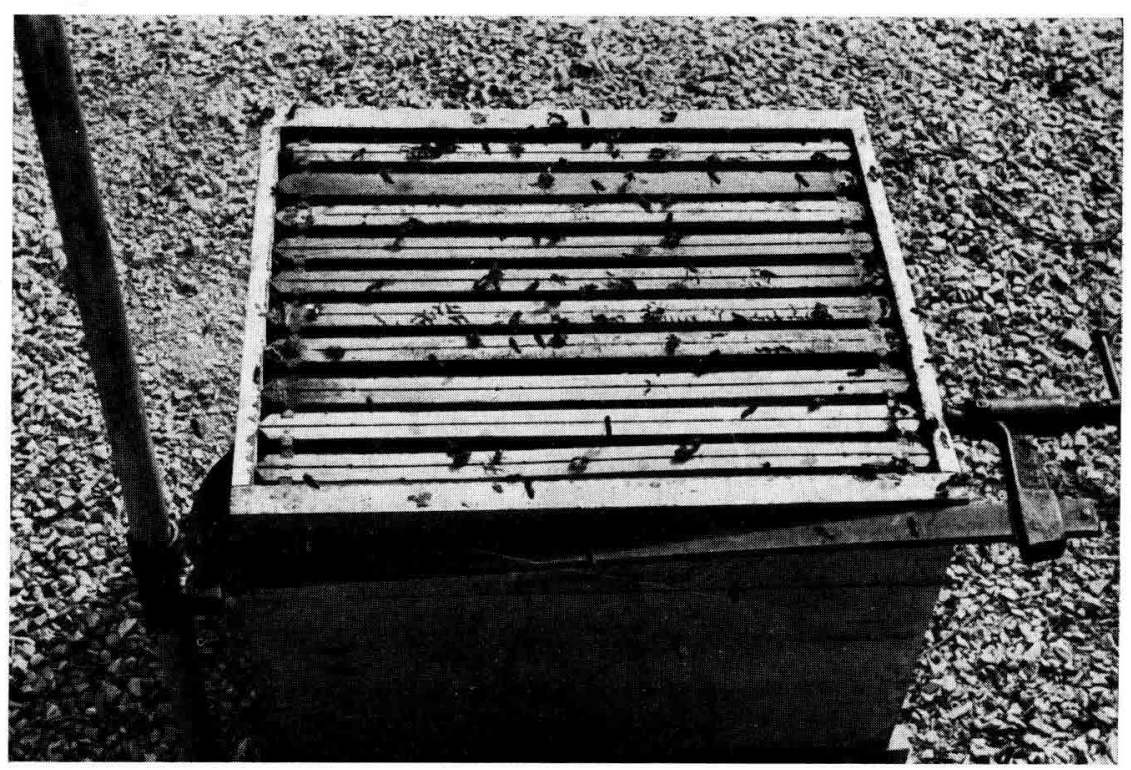

FIs. 3. - Le serre-joint 
İ́n dehors de la suppression du pillage et de l'agressivité des abeilles, on peut trouver d'autres utilisations de la cabine-parasol. Par exemple, empêcher que les reines vierges qui vont être inséminées artificiellement ne s'envolent avant d'être marquées et clippées.

L'utilisation de la cabine-parasol serait à conseiller également dans les pays oì les races d'abeilles sont très agressives, notamment au Maroc, en Afrique du Sud, ainsi que dans certains pays d'Amérique du Sud qui ont importé récemment des abeilles africaines (Apis adansoni) lesquelles se sont hybridées avec les abeilles locales en donnant des sujets particulièrement agressifs (NoGUEIRA-NETO, I963).

I.es résultats obtenus en utilisant la cabine-parasol nous permettent, par ailleurs, de confirmer les observations de L,ECOMTE ( I96I) en ce qui concerne les causes de 1'agressivité des abeilles. L'agressivité des abeilles dans les conditions habituelles des visites cles ruches est due principalement aux tentatives de pillage de la part des abeilles étrangètes à la ruche.

Il se peut également que les abeilles soient perturbées par la claustration relative et perdent, de ce fait, leur réflexe de défense.

\section{DISCUSSION}

L'utilisation de la cabine-parasol pour la visite des ruches supprime totalement 1e pillage et 1'agressivité des abeilles. Elle est simple à réaliser. Le montage et le démontage se font rapidement. L'encombrement de l'appareil plié est remarquablement faible. Les essais effectués montrent la pleine efficacité de ce dispositif, aussi bien en apiculture pratique qu'en apiculture expérimentale. I.a cabine-parasol est à conseiller particulièrement aux apiculteurs dont le cheptel comporte des abeilles très agressives, ou certaines races étrangères et en apiculture intensive oì certaines opérations sont longues, délicates et où le calendrier des travaux est fixé à l'avance.

Nos essais confirment que l'agressivité des abeilles pendant la visite des ruches est due à l'attaque des colonies visitées par les abeilles pillardes venues de ruches voisines.

Reçu pour publication en notembre $\mathbf{1}_{96} 3$.

\section{SUMMARY}

A DEVICE FOR TIE COMPLETE SUPPRESSION OF ROBBING AND AGGRESSIVENESS IN BEES IURING VISITS TO THl: HIVE

The description of an casily dismantled and transportable cage for the complete suppression of robbing and agressiveness in bees during visits to the hive. It consisted of a parasol and fine netting which formed a shelter preventing robber bees from attacking the hive. Its use was particularly recommended for the control of highly aggressive bees, trials having given entire satisfaction. Indications were that the aggressiveness shown by bees during the visit of the hive was caused very largely by attacks from robber bees arriving from neighlouring hives. 


\section{RÉFÉRENCES BIBLIOGRAPHIQUES}

Cinuuvin R., 1953. Le maintien de la ruche en milieu confiné est-il possible? L'Apiculteur Sect. Sci., 97 (1), 25-29.

Licomte J., 1955. Observations sur le comportement d'abeilles vivant en serres chaudes durant l'hiver. L'Apiculteur Sect. Sci., 9, 39-42.

Leсомте J., 1961. Le comportement agressif des ouvrières d'Apis mellifica I. Ann. Abeille, 4 (3), 165-270. NıQ̨uille O., 19'7. La mort de l'enfumoir. L'Apiculleur, 91 (1), 12-15.

Nogueira Neto P., 1963. Communication verbale.

Weber A., 19'3. Cité par Niquille. 